\title{
DU PARTAGE DE SAVOIRS INFORMELS AUX IDENTITÉS MÉDIATISÉES 1 Un dispositif de communication médiatisée par ordinateur : la page personnelle
}

\author{
Annabelle Klein ${ }^{2}$
}

Lors d'un précédent colloque organisé par le GReMS ${ }^{3}$, c'est la question du savoir et de ses médiations qui était posée de manière singulière à travers l'usage des nouvelles technologies.

Les nouvelles technologies, parce qu'elles sont susceptibles de s'inscrire dans une pluralité de champs sociaux, ont pu servir de marchepied à une appréhension plus transversale de la médiation des savoirs. L'inconvénient, toutefois, réside alors dans les réductions qu'une telle approche fait subir à la notion de "savoir": elle ramène le plus souvent ce dernier à une collection d'informations, négligeant par exemple ses dimensions relationnelle, pragmatique, affective ${ }^{4}$.

1 Cette communication s'inspire d'une recherche doctorale intitulée La présentation de soi sur Internet ou la page personnelle comme nouvelle figure de l'identité contemporaine. De l'identité narrative à l'identité médiatique.

2 Docteur en sciences sociales (information et communication), chercheur au GReMS (Groupe de Recherche en Médiation des Savoirs).

${ }^{3}$ Le GReMS organisait les 24 et 25 avril 1998 à Louvain-la-Neuve un colloque intitulé "Dispositifs \& Médiation des Savoirs".

4 Nous citons ici nos collègues, Philippe Charlier et Hugues Peeters, en reprenant un extrait de leur texte d'introduction au colloque en question. 
L'hypothèse soutenue à l'époque était celle d'un basculement de cette conception des savoirs comme contenus à une conception de la médiation des savoirs qui envisagent davantage l'expérience faite d'environnements aménagés, dans ses dimensions relationnelles et pragmatiques. Aujourd'hui, c'est la distinction et les articulations entre savoirs formels et savoirs informels qui est abordée.

Le dispositif particulier que représentent les pages personnelles permet de participer à l'affinement de cette hypothèse tout en abordant sa spécificité informelle. Il s'agit en effet d'envisager le phénomène de création d'une page personnelle sous l'angle d'une expérience particulière sur Internet où, bien sûr, différents types de savoirs (en tant que contenus ou informations) circulent, s'échangent, se co-construisent mais où la dimension expressive, relationnelle et pragmatique est également prise en compte.

En outre, et au même titre que les forums par exemple, les pages personnelles constituent de véritables lieux de savoirs informels à partir desquels s'inscrit de la relation et s'ébauchent des identités médiatisées ${ }^{1}$. A titre d'illustration, reprenons quelques extraits d'entretiens menés par mails :

"Tu devrais prendre contact avec $\mathrm{A}$, elle a réalisé un excellent site sur les journaux intimes sur Internet", "B, c'est le site très généreux des cartes postales", etc.

Le créateur d'une page devient alors expert, c'est-à-dire détenteur d'une ressource socialement utile, d'un savoir informel.

C'est ainsi que l'introduction des nouvelles technologies ouvre un nouveau champ d'expression de soi et de participation à l'élaboration de savoirs. En effet, parmi les multiples usages d'Internet, la création de dispositifs de mise en forme de soi prend une place de plus en plus importante. Ceux-ci, nommés diversément pages personnelles, homepages, sites web personnels ou encore pages perso, offrent à chacun l'espace d'une mise en scène de soi, de ses activités, de ses passions, de ses projets, de son histoire personnelle, etc. Ces sites web d'individus constituent des espaces électroniques de présentation de soi et l'on peut s'interroger sur l'éventuel caractère formateur de cette nouvelle pratique. En effet, ces constructions multimédiatiques (écrits, images fixes ou animées, photographies, son, musique, etc.) ont pour caractéristique de créer des récits de soi

1 Qui dépassent le plus souvent le seul cadre des pages personnelles. 
contemporains qui permettent à chacun de "se donner forme". Et ce, tant à travers la création/écriture/production de son site personnel qu'à travers la découverte/lecture/consommation d'autres pages personnelles.

Cet article ${ }^{1}$ propose de découvrir d'une part, en quoi ces nouvelles activités d'écriture/lecture de soi et de l'autre sont formatrices au sens d'une médiatisation et d'une mise en forme identitaire et d'autre part, en quoi elles redéfinissent les rapports entre savoirs formels et savoirs informels en constituant des nouveaux lieux de partage de savoirs informels.

\section{En quoi la réalisation d'une page personnelle peut-elle être formatrice?}

L'idée qui s'est progressivement dégagée de notre recherche consiste à penser qu'il est possible d'envisager les pages personnelles autrement que comme de simples curriculum vitae ou comme des activités purement ludiques. En effet, contrairement à d'autres formes et espaces de communication interpersonnelle cadrés par Internet qui se limitent au transfert d'usages communicationnels existants déjà socialement, les pages personnelles constituent des pratiques innovantes qui sont à la fois créatrices et formatrices.

Créatrices, d'abord, au sens où, en tant qu'espaces d'autopromotion et d'auto-définition, les pages personnelles n'ont pas de véritable équivalent social ${ }^{2}$, contrairement à d'autres activités internautiques comme le mail, le chat, le forum, etc., qui se réfèrent plus directement à des activités communicationnelles telles que la correspondance écrite, la discussion, le colloque ou le symposium, pour n'en citer que quelques-uns.

Les formes actuelles de communication interpersonnelle sur Internet apparaissent peu innovantes (les avatars et les mondes virtuels sont encore trop rares pour en parler en termes d'usages) et surtout guère plus avantageuses que leurs

1 D'un point de vue méthodologique, nous avons souhaité nous centrer exclusivement sur l'analyse de pages personnelles telles qu'elles se présentent sur le réseau, tout en y adjoignant certains extraits pertinents d'entretiens témoignant de l'expérience identitaire à l'œuvre à travers l'acte de lecture/écriture et, plus largement, de création/visites de pages personnelles sur Internet.

2 Au sens où il s'agit d'une "vitrine de soi", d'un véritable espace publicitaire prenant la personne elle-même comme objet. 
équivalents non informatiques : seule la page web représente sans doute une ouverture publicitaire individuelle dont l'équivalent reste difficile à trouver' ${ }^{1}$.

Les pages personnelles sont également créatrices au sens où elles rendent leurs auteurs libres de différentes contraintes (physiques, sociales, culturelles, etc.). Dans son ouvrage intitulé Stigmates ${ }^{2}$, Erwing Goffman définit l'identité sociale virtuelle comme étant celle que nous attendons d'une personne ou que le social attend d'elle tandis que l'identité réelle est celle que cette personne peut réellement offrir. Le stigmate, quant à lui, est l'attribut qui rend l'individu différent de la catégorie qui lui est ouverte, c'est-à-dire lorsqu'il y a désaccord entre l'identité réelle et l'identité virtuelle, lorsqu'il y a cassure entre soi et ce qu'on exige de soi. Goffman souligne le fait que toute interaction sociale fait l'objet d'une définition, c'est-à-dire d'un modus vivendi interactionnel qui tend à régler le déroulement de la coopération et qui présente toujours un indéniable caractère moral. Les deux principes définis plus haut qui sous-tendent cette définition sont donc d'une part, que toute personne possédant certaines caractéristiques sociales est moralement en droit d'attendre qu'on l'estime et d'autre part, que si quelqu'un prétend avoir certaines caractéristiques sociales, on attend de lui qu'il soit réellement ce qu'il prétend être. Pourtant, la scène des pages personnelles échappe pour une large part à ces attentes communicationnelles puisque précisément des possibilités de non-adéquation entre la représentation de soi et ses caractéristiques biographiques, sociales et autres font partie du jeu communicationnel. Il est alors possible d'expérimenter d'autres façons de se présenter, de se raconter, et donc d'autres modes d'être. Ainsi, cette jeune fille qui invente la page personnelle d'un homme, se mettant ainsi dans la peau du sexe opposé, ce chercheur d'emploi qui scinde sa page perso en plusieurs parties, adressées à des publics différents et dévoilant des facettes différenciées de lui-même, ou encore ce jeune garçon d'à peine douze ans qui construit sa page autour d'une passion -l'aviation- de manière si convainquante, perfectionnée et documentée que ses visiteurs l'imaginent régulièrement avec une longue carrière de pilote derrière lui !

1 D. Boullier et C. Charlier, "A chacun son Internet. Enquête sur des usagers ordinaires", Réseaux, n 86, CNET, 1997, p. 174.

2 E. GofFMAN Stigmates. Les usages sociaux des handicaps, Paris, Éd. de Minuit, coll. "Le sens commun", 1963, p. 12. 
Avec Internet donc, le stigmate, à savoir l'écart entre ce que l'on est (l'identité réelle) et ce que l'on attend de nous (l'identité sociale virtuelle), se trouve redéfini puisque les auteurs de pages personnelles sont libérés de tous ces signes patents ou porte-identité, c'est-à-dire la combinaison unique de faits biographiques qui finit par s'attacher à l'individu, cet "enregistrement unique et ininterrompu de faits sociaux qui vient s'attacher, s'entortiller, comme de la barbe-à-papa, comme une substance poisseuse à laquelle se collent sans cesse de nouveaux détails biographiques"1.

La page personnelle de Jean-Pascal est exemplaire à cet égard. À notre étonnement et notre questionnement face à l'absence totale d'éléments biographiques le concernant, il répond : " $C$ 'est sans doute là l'un des intérêts d'Internet : il nous libère de tout cela et permet ainsi une espèce de rencontre des esprits".

Lors de nos entretiens par mail, MöngôlO ${ }^{2}$ nous dit sortir des contraintes de la vie quotidienne pour exister autrement, pour exprimer ce qui est inexprimable ailleurs...

Faire sa page personnelle, c'est une façon de se prendre et de se mettre en commun. C'est un peu comme si l'existence dans la vie de tous les jours était trop restreignante pour exister de la façon dont on voudrait exister. Mettre sa page perso en ligne permet de changer cette existence et d'exprimer des choses inexprimables dans la vie ordinaire. Soit parce que ces choses auraient trop de répercussions, soit parce que personne est là pour les écouter, soit parce que l'expression elle-même est impossible dans la vie ordinaire, soit parce que cette expression a besoin d'un support que le monde online rend accessible $^{3}$.

Ceci permet, d'une part, une expression singulière moins marquée par les multiples appartenances, et d'autre part, une place indéniable donnée à la fiction, rendant dès lors la personne qui s'y prête susceptible de devenir un véritable personnage, ouvert à toutes les inventions biographiques. Il est intéressant de réfléchir à la gamme de possibilités offertes par ces nouveaux médias à repousser les

1 Ibid., p. 74.

2 Qui est, le lecteur l'aura deviné, une espèce de nom de scène, communément appelé nickname, qu'un internaute choisit d'utiliser tantôt pour s'identifier dans le cadre de chats ou de forums, tantôt pour construire sa page personnelle autour d'un personnage.

3 Extrait de sa page personnelle, intitulée Möngôlo's Diary (almost) aujourd'hui disparue. 
limites et la confrontation au réel. Tout peut être réinventé. Cette scène virtuelle ne permet-elle pas en effet de créer de toutes pièces un personnage, pour jouer, pour réaliser celui ou celle que nous voudrions être, pour inventer une autre vie? La page personnelle deviendrait-elle un terrain d'essai, essai à devenir soi, où s'essayer à être?

Créatrice donc, la homepage est aussi formatrice et cette affirmation exige un développement en cinq points.

\section{La page personnelle, une ouverture entre auteur et visiteur}

Premièrement, elle aide à penser les modifications profondes du rapport entre auteur et visiteur. On assiste en effet sur Internet à une intrication de ces pôles communicationnels. Citons, à titre d'exemple, les romans collectifs qui naissent actuellement sur la toile : le principe consiste à participer à sa réalisation en complètant ce que l'internaute précédent a apporté à l'œuvre commune. On assiste alors à une démultiplication de la notion d'auteur et à un processus d'énonciation multiple. De même, et aussi étonnant que cela puisse paraître puisqu'elles sont des productions d'auteur par excellence, les pages personnelles introduisent l'Autre pour se définir. En effet, elles doivent être envisagées en tant qu'elles engagent une mise à distance de soi puisqu'on se "place" ailleurs ; on se retrouve peu ou prou "face" à soi-même, notamment à travers les yeux des autres sur soi. Des processus de centration et de décentration, d'identification et de désidentification opèrent et se renvoient les uns aux autres dans une dynamique incessante. Ce mouvement de rapport avec soi, de dialogue avec soi à travers les autres, s'atteste à travers les marques d'adresse explicites à l'autre ("Qu'en pensez-vous ?", "Etes-vous d'accord avec moi ?", "Laissez-moi un commentaire", ou encore "Signez mon livre d'or pour laisser une trace de votre passage" ou encore "Bientôt, un diagnostic complet de ma personne, grâce à vous, peut-être ?"). Certaines pages personnelles sont d'ailleurs construites à travers ce "multilogue" en rapatriant au sein de la page elle-même, tout en les mettant en perspective, les avis et commentaires divers des internautes passés par là. Mais à qui s'adresse-t-on, au juste ?

Dans certains cas, il s'agit de s'adresser à tout internaute potentiel, pourvu qu'il ne puisse s'établir de ponts avec d'autres sphères 
identitaires de la vie quotidienne. MôngölO illustre parfaitement cette position en annonçant: "Si vous me connaissez, passez votre chemin... car cette page n'est pas destinée à être lue par des personnes que je connais..." D'autres pages personnelles sont, à l'inverse, adressées avant tout à des personnes connues, plus ou moins proches, constituant un réseau ou même à une seule personne, instituant ainsi un lieu de communication et de présentation de "son monde" à l'autre, choisi et seul détenteur de l'adresse du site personnel'.

Dans tous les cas, la création d'une page personnelle constitue une façon de se dire et de poser la question de son identité à travers le détour de l'autre. Il s'agit d'introduire de l'altérité dans la construction identitaire, ce qui rejoint le terme ricoeurien d'ipse, qui signifie l'autre dans le même. Cette intrusion de l'altérité au coeur du procès identitaire rejoint le constat déjà évoqué d'une mutation pragmatique de l'énonciation :

De ce fait, la notion d'auteur se dissout, de même que celle de lecteur qui se rapproche de celle de contributeur et par là de celle d'auteur (ce qu'elle a toujours été d'une certaine façon mais qui ici s'instrumente) ${ }^{2}$.

On assiste ici à un partage de "responsabilité éditoriale" : chacun peut participer à l'élaboration d'un savoir, fut-il informel ${ }^{3}$. Les homepages sont dès lors formatrices au sens où, en partageant l'énonciation et en rendant ainsi visible la place de l'Autre dans son propre discours, se développe et s'incarne dans ce que Jean Piaget appelle la décentration, c'est-à-dire cette capacité à prendre en compte plusieurs points de vue pour construire le sien. Il s'agit donc d'envisager la communication identitaire à travers un travail d'ouverture à l'autre. Cette ouverture est particulièrement présente d'une part dans l'expérience de création et de visite de pages personnelles puisque son créateur s'adresse le plus souvent à un public ouvert, indéterminé et multiple et d'autre part, dans ce que Marc Lits appelle un éclatement

1 Cette posture est nettement plus rare néanmoins.

2 D. Boullier et C. CARLIER, op. cit., p. 173.

3 Nous y reviendrons au point suivant. 
énonciatif $^{1}$. En effet, si le destinataire est démultiplié et indéterminé ${ }^{2}$, l'émetteur lui-même n'est plus unique ni unifié dans son énonciation ${ }^{3}$. Car, malgré le fait qu'une page personnelle porte le nom ou le surnom de son auteur et constitue un lieu identitaire de présentation de soi sur Internet, l'émetteur ne se construit que dans la relation avec ses récepteurs puisqu'il les sollicite à le compléter, à lui donner d'autres idées, à lui dire qui il est, en réinjectant parfois ces commentaires dans la page de façon circulaire. C'est donc à une mutation profonde du mode d'énonciation et, plus largement, de la pragmatique de la communication que nous avons affaire.

Face à cette discursivité circulaire, nous pourrions partager avec Marc Lits l'idée que cette démultiplication énonciative engendre une perte identitaire. Nous pourrions encore le suivre lorsqu'il annonce que "Le risque d'Internet n'est pas celui de la mort du sujet, mais de sa dissolution dans trop de sujets"4, s'il n'y avait ces multiples formes narratives, à notre sens toutes créatrices d'identités. Associer discursivité circulaire et démultiplication énonciative à une perte identitaire ne nous semble pas automatique. Car c'est sans compter sur la reprise de cette diversité énonciative en narration. Celle-ci implique des processus de configuration dont la homepage semble consituer un lieu privilégié. Nous l'envisageons comme un espace d'auto-présentation où la place de l'auteur est donc, en dernier ressort, sauvegardée. Ces formes narratives restaurent, malgré la diversité énonciative dont nous parlions, des identités énonciatives. Nous approfondirons la question de la configuration et des formes narratives dans le dernier point ${ }^{5}$.

\section{La page personnelle en partage de savoirs informels}

Deuxièmement, si l'on envisage la formation en termes d'acquisition de savoirs, on peut dire que tant la visite que la création de

1 M. LITs, "Vers un récit multimédiatique ?", Médiatiques. Récit et société $\mathrm{n}^{\circ} 20$, ORM, Printemps 2000, p. 49.

2 La homepage peut s'adresser sélectivement ou conjointement à n'importe quel quidam, à l'internaute inconnu et lointain, à un groupe d'internautes connus dans d'autres cadres (chats, mails, etc.), à sa famille, à des amis, etc.

3 En fait, il ne l'est jamais mais, une fois encore, le dispositif communicationnel appuie, prolonge et incarne cet état de fait.

4 M. LITs, op. cit., p. 50.

5 Cf. La homepage, une prise de forme identitaire. 
pages personnelles sont des actes formateurs car les savoirs qui y circulent sont d'ordre divers : savoirs techniques (informatique, le plus souvent), savoirs médiatiques (cinématographiques, par exemple), savoirs culturels (présentations et/ou critiques d'événements culturels, partage de commentaires ou d'extraits musicaux, etc.), savoirs culinaires mais aussi "cyberculturels" ("comment j'ai fait pour entrer dans tel ou tel forum", trucs et ficelles technologiques), savoirs existentiels, savoirs de conscientisation, bref, savoirs divers et surtout, savoirs sur soi, savoirs biographiques en quelque sorte. On peut parler d'un partage de savoirs -en tant que contenusen tous genres. On pourrait dire que tous ces savoirs ont pour principale caractéristique d'avoir été préalablement pétris d'expérience par ceux qui en font état.

Ce partage de savoirs informels -informels au sens où ils ne retirent leur légitimité que de l'expérience et de l'appropriation par l'auteur- est formateur par l'échange qu'il permet d'expériences portant sur des contenus divers. Chacun peut ainsi partager son savoir sur la scène publique. Cette position est même parfois adoptée à l'extrême par les créateurs de homepages. Ainsi Jean-Pascal sollicitet-il constamment ses visiteurs à l'imiter en partageant ce qu'ils savent. Lors de l'un de nos entretiens par mail, il nous écrira : "Ma principale motivation en faisant cela, c'est : je connais quelques petites choses, je me dois d'offrir cette «compétence» aux autres. Je harcèle souvent mes visiteurs pour qu'ils fassent leur propre page ! C'est mon côté instituteur ;-)" ou encore "chacun se doit de partager ce qu'il sait, et Internet en est le meilleur moyen. On ne peut pas toujours prendre sans rien donner, quoi !". Ainsi, il nous semble que se généralise un nouveau rapport au savoir, qu'il s'agisse de savoirs scientifiques, historiques, informatiques, etc., ou de savoirs-être, de savoir sur soi, de savoirs d'expérience de vie.

Nous l'avons annoncé en introduction, ces savoirs informels ne doivent pas être envisagés exclusivement comme des contenus (information) mais également en termes relationnels (communication). Après avoir présenté la première partie du titre de cette communication, à savoir l'idée de savoirs informels, attelonsnous à l'idée de partage et de co-construction de ces savoirs. 


\section{La page personnelle, lieu ou espace de soi ?}

D'emblée, il est important de distinguer l'objet et le dispositif "page personelle". L'objet "page personnelle", c'est la page ellemême hic et nunc, c'est-à-dire la configuration émergeant de la mise en liaison de multiples parcelles de soi à travers les liens, l'agencement des contenus, le style emprunté, etc. Il ne s'agit pourtant là que de la part visible de l'iceberg que constitue le dispositifhomepage. Ce dernier comprend les visiteurs et interlocuteurs potentiels ou visés explicitement et donc inclut la mise à disposition de soi sur le net, les échanges, les changements et transformations dans la présentation de soi, les actes et traces de visites, de mises en lien avec d'autres pages personnelles, etc. Le dispositif-homepage, c'est donc encore ce qui en est fait lorsque, telle une ouvre qui s'expose et devient partagée, elle échappe à son créateur, à son auteur pour devenir ce que d'autres en feront.

Une seconde distinction -que nous pensons en partie couplée à la première- est celle qu'établit Michel de Certeau au sujet du lieu et de l'espace. S'inspirant de la perspective phénoménologique de MerleauPonty qui déjà distinguait l'espace géométrique de l'espace anthropologique, Michel de Certeau définit le lieu comme :

Un ordre selon lequel des éléments sont distribués dans des rapports de coexistence. S'y trouve donc exclue la possibilité, pour deux choses, d'être à la même place. La loi du "propre" y règne : les éléments considérés sont les uns à côté des autres, chacun situé en un endroit "propre" et distinct qu'il définit. Un lieu est donc une configuration instantanée de positions. Il implique une indication de stabilitél.

L'espace, quant à lui, serait au contraire un croisement de mobiles.

Il est en quelque sorte animé par les mouvements qui s'y déployent. Est espace l'effet produit par les opérations qui l'orientent, le circonstancient, le temporalisent et l'amènent à fonctionner en unité polyvalente de programmes conflictuels ou de proximités contractuelles... A la différence du lieu, il n'a donc ni univocité ni stabilité d'un "propre". L'espace serait au

1 M. DE Certeau, L'invention du quotidien. Arts de faire, Paris, Union générale d'éditions, 1980, p. 208. 
lieu ce que devient le mot lorsqu'il est parlé, c'est-à-dire quand il est saisi dans l'ambiguité d'une effectuation, mu en un terme relevant de multiples conventions, posé comme l'acte d'un présent (ou d'un temps), et modifié par les transformations dues à des voisinages successifs ${ }^{1}$.

Prenons d'abord un exemple issu de l'espace construit matériellement pour mieux comprendre cette distinction et ensuite illustrer plus spécifiquement en quoi les concepts de lieu et d'espace permettent de différencier un double plan au sein des pages personnelles.

Le lieu que constitue une maison se trouve transformé en espace par ses occupants dès l'instant où, habitée, elle devient en quelque sorte "pratiquée". Sortant alors de l'ordre univoque et de la configuration stable et instantanée, elle devient effective, habitée, pratiquée et ainsi variable, mobile, plurivoque, toujours en mouvement, en devenir. En somme, disons que l'espace est un lieu qui est pratiqué.

Comme pour la maison, on peut en effet parler de lieu pour désigner la homepage en tant que site, point de repère personnel, aire de référence identitaire spécifique à la sphère Internet, position inscrite dans l'ordre des places que tisse le web. Ce lieu propre porte un nom, une adresse, et situe celui qui y dépose une part de lui à une place définie sur cette toile du web.

Par ailleurs, tout l'intérêt des pages personnelles réside dans la transformation de ce lieu en espace de soi, c'est-à-dire dans sa mise en pratique, dans son "occupation". Ainsi, la visite d'une page personnelle par un internaute en fait un espace, un lieu "pratiqué", "expérimenté", que ce visiteur soit d'ailleurs connu ou inconnu de son auteur, ou encore qu'il s'agisse de l'auteur lui-même. Car celui-ci se visite régulièrement pour y rencontrer cet autre de lui-même, cette part de soi et la modifier, la réduire, l'agrandir, la transformer, l'embellir, la supprimer, etc.

Ce qui constitue l'une des caractéristiques du phénomène étudié, c'est que le lieu de soi porté par une homepage est en effet dénué de sens s'il n'est pas transformé en espace de soi, c'est à dire si les savoirs informels ne sont pas échangés, partagés, co-construits ${ }^{2}$. Ce

1 Ibid., p. 208.

2 Tout nous pousse à penser que les récits de soi sur Internet, à travers le support médiatique que constituent les pages personnelles, se transforment au contraire en espaces. Ainsi, à partir des pages personnelles telles qu'elles se présentent sur la toile, il nous est possible de repérer les types d'adresses à l'autre, les transformations temporelles émanant ou non des interactions entre internautes, les 
qui nous pousse à avancer qu'une page personnelle qui ne serait jamais visitée demeurerait lettre morte, condamnée à rester lieu, dans toute sa fixité et sa stabilité.

\section{La page personnelle, de la reprise de l'expérience à la création d'un "cybersoi"}

Quatrièmement, la page personnelle, comme lieu et espace de soi, a pour particularité de constituer une aire de reprise de l'expérience -par rapatriement d'autres sphères identitaires-qui produit une nouvelle expérience spécifique. Celle de "se mettre en commun" et de créer une entité spécifique, pour reprendre les termes de notre ami MöngôlO :

A la limite, l'écriture du journal en ligne s'apparente à un exercice de schizophrène où une partie réelle de soi se projette sous une autre forme pour entrer dans un milieu. Par exemple, à l'origine MöngôlO était seulement un pseudonyme destiné à empêcher les personnes que je connaissais de me reconnaître parce que ce journal leur était pas adressé. Mais petit à petit, MöngôlO est devenu une vraie entité, probablement au moins légèrement différente de l'entité qui la projette. Tout est séparé chez moi, l'endroit où les mails arrivent, ma façon de répondre, le contenu de mes réponses, même la langue utilisée pour la réponse... Pourtant c'est bien la même personne qui répond pour moi et pour Möngôlo. Mine de rien, et sans en avoir vraiment la volonté, j'ai créé une nouvelle entité, probablement une façette de moi-même qui s'exprimait pas avant.

Cette citation montre assez bien la spécificité de l'expérience identitaire qu'engendre la création d'un journal sur Internet ou, plus largement, d'une page personnelle. Elle incite à percevoir l'importance de l'autonomisation de ce lieu par rapport à d'autres afin que cette figure de soi (et sa mise en commun sur le net) puisse se détacher du reste de la vie quotidienne pour dépasser les procès de reprise d'expérience et faire émerger une nouvelle façon de se dire. C'est en cela que nous pensons que les pages personnelles s'avèrent parfois de véritables lieux/espaces suffisamment protecteurs pour la création de nouvelles formes identitaires. La homepage devient alors formatrice

marques de présence du destinataire, réel ou potentiel, etc. Toutes ces traces structurent et supportent la construction de l'objet-homepage (lieu) tout en en dévoilant le dispositif sous-jacent (espace). 
d'identités propres au média utilisé et jusqu'alors inédites. C'est ce que nous avons appelé l'émergence d'un "cybermoi".

\section{La page personnelle, une mise en intrigue de soi ?}

Cinquièmement, l'axe formateur, à nos yeux le plus important, qui est en jeu dans les pages personnelles est lié à l'occasion d'une "prise de forme identitaire" à partir des différents savoirs informels véhiculés et partagés. Ne permettent-ils pas avant tout de se situer dans le monde et de se réhistoriciser, c'est-à-dire de se présenter sous une forme narrative ? La homepage offre à l'individu contemporain non seulement un lieu de présentation de soi et de partage de savoirs informels mais aussi, et peut-être surtout, un espace d'articulation, de mise en forme et en sens de ses fragments identitaires épars. Car s'il est vrai qu'Internet offre d'autres lieux qui ouvrent à l'expression de soi et interrogent la question de l'identité, l'une des spécificités des "homepages" -en opposition avec d'autres espaces de communication sur Internet- est fondée sur leur caractère profondément configurant et narratif. Et ceci n'est évidemment pas étranger au fait qu'il s'agit, contrairement à tous ces espaces internautiques qui s'effectuent en direct, d'un espace de communication asynchrone. La homepage se distingue en effet d'un grand nombre d'activités de communication menées via Internet comme par exemple les chats ou les forums précisément par son exigence de configuration et de mise en intrigue, voire de scénarisation du personnage principal, l'auteur. Aussi envisageonsnous les pages personnelles comme des configurations de soi. Plus particulièrement, développons en quoi les homepages, envisagées comme constructions narratives et configurations de soi, participent à l'élaboration identitaire de façon privilégiée et spécifique.

La narration constitue le "genre communicationnel" le plus approprié à la construction identitaire car, ainsi que Paul Ricœur l'a magistralement développé, la mise en forme et la mise en intrigue de soi par la narration contribue à notre positionnement identitaire face aux autres et au monde qui nous entoure. En d'autres termes, la narration de soi est ainsi placée au coeur de la problématique identitaire en ce qu'elle permet une mise en scène et en intrigue de soi. La page personnelle constitue une configuration, au sens de mise en forme et en intrigue d'éléments épars de soi et de transformation éventuelle des événements en une histoire. Bref, il s'agit de l'organisation dans une 
totalité intelligible et signifiante d'une énumération de traits distinctifs ou définitoires, de passions, d'activités, d'événements, de pensées, de savoirs, etc. "Je vois dans les intrigues que nous inventons le moyen privilégié par lequel nous configurons notre expérience temporelle confuse, informe et, à la limite, muette"1.

La homepage peut devenir intrigue de soi, espace d'historisation des fragments identitaires disséminés. Ce cadre de promotion de soi sur la scène publique a pour conséquence de permettre le développement des capacités narratives et de créativité vis-à-vis de soi. Encore faudrait-il spécifier comment l'objet et le dispositif d'une page personnelle concourent à la création d'un nouveau type de narration, mais cela nous mènerait bien au delà des préoccupations de cet article consacré à la fonction jouée par les nouvelles technologies dans les mutations entre savoirs formels et savoirs informels.

\section{Conclusions}

A travers une argumentation en cinq points, nous avons tenté de montrer 1) en quoi les pages personnelles sont identitairement et communicationnellement créatrices, novatrices et surtout, formatrices au sens d'une mise en forme identitaire et 2) en quoi elles redéfinissent les rapports entre savoirs formels et savoirs informels en constituant des nouveaux lieux de partage de savoirs informels. Face à ce nouveau rapport au savoir, deux postures peuvent être adoptées : soit, il n'y a plus de savoirs formels ${ }^{2}$ sur Internet car les procédés de légitimation sont flous et mouvants, voire inexistants pour bon nombre d'usagers, soit, tout devient savoir formel car chacun devient expert d'un savoir légitimé par lui-même. À travers ces deux positions extrêmes, induites par les nouvelles technologies, on perçoit aisément que l'on assiste à un écrasement des deux notions -savoirs formels, savoirs informels- qui entache sérieusement leur pertinence conceptuelle.

1 P. RICEUUR, Temps et récit, Paris, Éd. du Seuil, Tome 1, 1983, p. 106.

2 C'est précisément cette situation qui pose tant de problèmes et de craintes aux enseignants, parents et éducateurs au sens large. Comment maintenir une prise de distance et un esprit critique face à une énonciation multiple et à des sources tout aussi démultipliées et non situées ? Sans doute s'agit-il de développer un nouveau type d'esprit critique qui tiendrait compte de ce nouveau contexte d'énonciation du savoir. 